\title{
Tropospheric temperature measurements with the pure rotational Raman lidar technique using nonlinear calibration functions
}

\author{
Vladimir V. Zuev ${ }^{1,2,3}$, Vladislav V. Gerasimov ${ }^{1,2}$, Vladimir L. Pravdin ${ }^{1}$, Aleksei V. Pavlinskiy ${ }^{1}$, and \\ Daria P. Nakhtigalova ${ }^{1}$ \\ ${ }^{1}$ Institute of Monitoring of Climatic and Ecological Systems SB RAS, Tomsk, 634055, Russia \\ ${ }^{2}$ Tomsk State University, Tomsk, 634050, Russia \\ ${ }^{3}$ Tomsk Polytechnic University, Tomsk, 634050, Russia \\ Correspondence to: Vladislav V. Gerasimov (gvvsnake@mail.ru)
}

Received: 1 June 2016 - Published in Atmos. Meas. Tech. Discuss.: 13 June 2016

Revised: 29 December 2016 - Accepted: 2 January 2017 - Published: 27 January 2017

\begin{abstract}
Among lidar techniques, the pure rotational Raman (PRR) technique is the best suited for tropospheric and lower stratospheric temperature measurements. Calibration functions are required for the PRR technique to retrieve temperature profiles from lidar remote sensing data. Both temperature retrieval accuracy and number of calibration coefficients depend on the selected function. The commonly used calibration function (linear in reciprocal temperature $1 / T$ with two calibration coefficients) ignores all types of broadening of individual PRR lines of atmospheric $\mathrm{N}_{2}$ and $\mathrm{O}_{2}$ molecules. However, the collisional (pressure) broadening dominates over other types of broadening of PRR lines in the troposphere and can differently affect the accuracy of tropospheric temperature measurements depending on the PRR lidar system. We recently derived the calibration function in the general analytical form that takes into account the collisional broadening of all $\mathrm{N}_{2}$ and $\mathrm{O}_{2}$ PRR lines (Gerasimov and Zuev, 2016). This general calibration function represents an infinite series and, therefore, cannot be directly used in the temperature retrieval algorithm. For this reason, its four simplest special cases (calibration functions nonlinear in $1 / T$ with three calibration coefficients), two of which have not been suggested before, were considered and analyzed. All the special cases take the collisional PRR lines broadening into account in varying degrees and the best function among them was determined via simulation. In this paper, we use the special cases to retrieve tropospheric temperature from real PRR lidar data. The calibration function best suited for tropospheric temperature retrievals is determined from the comparative analysis of temperature uncer-
\end{abstract}

tainties yielded by using these functions. The absolute and relative statistical uncertainties of temperature retrieval are given in an analytical form assuming Poisson statistics of photon counting. The vertical tropospheric temperature profiles, retrieved from nighttime lidar measurements in Tomsk $\left(56.48^{\circ} \mathrm{N}, 85.05^{\circ} \mathrm{E}\right.$; Western Siberia, Russia) on 2 October 2014 and 1 April 2015, are presented as an example of the calibration functions application. The measurements were performed using a PRR lidar designed in the Institute of Monitoring of Climatic and Ecological Systems of the Siberian Branch of the Russian Academy of Sciences for tropospheric temperature measurements.

\section{Introduction}

The pure rotational Raman (PRR) technique is known to be the best suited for lower atmosphere temperature measurements (Wulfmeyer et al., 2015). The retrieval algorithm of vertical temperature profiles of the troposphere and lower stratosphere from PRR lidar raw signals consists of four main steps:

- PRR lidar raw data averaging to improve the signal-tonoise ratio and decrease the statistical uncertainties;

- lidar calibration, i.e., determination of the lidar calibration function coefficients by applying, for example, the least square method to the reference radiosonde (or model) data and previously averaged lidar data; 
- temperature profile retrieval by using the temperature retrieval function derived from the selected calibration function;

- estimation of the absolute and relative uncertainties of the temperature retrieval and calculation of the difference between the reference temperature (radiosonde, model) and temperature retrieved from lidar data.

The PRR lidar technique suggested by Cooney (1972) is based on the temperature dependence of individual lines intensity of atmospheric $\mathrm{N}_{2}$ and $\mathrm{O}_{2}$ PRR spectra. The intensity $I(T, \lambda)$ of a single PRR line of the wavelength $\lambda$ backscattered by excited $\mathrm{N}_{2}$ or $\mathrm{O}_{2}$ molecules can be expressed as (Penney et al., 1974)

$I(\lambda, T)=P L \beta_{\pi}(\lambda, T)$,

where $P$ is the incident laser-beam power, $L$ is the length of the scattering volume, and $\beta_{\pi}(\lambda, T)$ is the backscatter cross section (atmospheric backscatter coefficient). The backscattered signals of the Stokes and/or anti-Stokes branches of the spectra can be used for temperature determination. The intensities of individual PRR lines, corresponding to low and high rotational quantum numbers $J$ of the initial states of the PRR transitions, are of opposite temperature dependence (Behrendt, 2005). Namely, the intensity of each $\mathrm{N}_{2}$ PRR line with $J_{\text {low }} \leq 8\left(J_{\text {low }} \leq 9\right.$ for $\mathrm{O}_{2}$ PRR lines $)$ decreases with increasing temperature and, conversely, the intensity of $\mathrm{N}_{2}$ PRR lines with $J_{\text {high }} \geq 9\left(J_{\text {high }} \geq 11\right.$ for $\mathrm{O}_{2}$ PRR lines $)$ increases with increasing temperature in both branches of the spectra (Fig. 1). Note that only odd lines beginning with odd $J$ exist in $\mathrm{O}_{2}$ PRR spectrum (Wandinger, 2005). A ratio of backscattered signal intensities from two PRR-spectrum bands with opposite temperature dependence is required for air temperature $T$ determination. However, the PRR lidar theory (Cooney, 1972) gives the exact temperature dependence only for intensity ratios of two individual PRR lines corresponding to certain $J_{\text {low }}$ and $J_{\text {high }}$

$Q^{\text {indiv. }}(T)=\frac{I\left(J_{\text {low }}, T\right)}{I\left(J_{\text {high }}, T\right)}=\frac{\beta_{\pi}\left(J_{\text {low }}, T\right)}{\beta_{\pi}\left(J_{\text {high }}, T\right)}=\exp \left(\alpha+\frac{\beta}{T}\right)$,

where the constants $\alpha$ and $\beta$ are completely defined from the theory.

In practice, diffraction gratings (DGs) or interference filters (IFs) extract several adjacent PRR lines in the lidar temperature channels from backscattered light. IFs extract PRR lines from the anti-Stokes branches of $\mathrm{N}_{2}$ and $\mathrm{O}_{2}$ PRR spectra (Behrendt and Reichardt, 2000; Behrendt et al., 2002, 2015; Alpers et al., 2004; Di Girolamo et al., 2004; Radlach et al., 2008; Achtert et al., 2013; Newsom et al., 2013; Li et al., 2015). DGs extract PRR lines from both the Stokes and anti-Stokes branches of the spectra (Ansmann et al., 1999; Kim et al., 2001; Chen et al., 2011; Jia and Yi, 2014). Thus, one should consider the following expression (Arshinov et

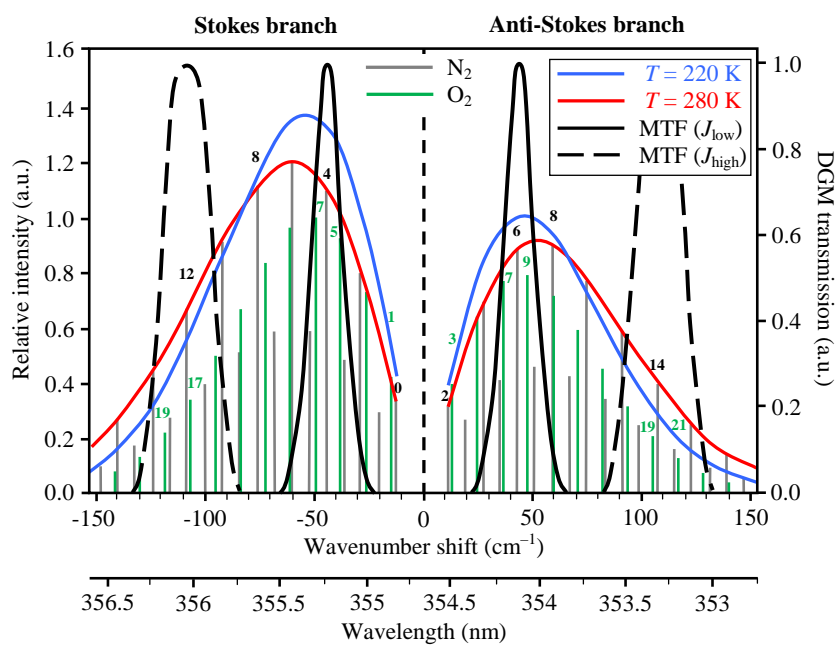

Figure 1. Equidistant PRR spectra of $\mathrm{N}_{2}$ and $\mathrm{O}_{2}$ linear molecules, schematic drawing of the IMCES lidar monochromator transmission functions (MTF), and envelopes of $\mathrm{N}_{2}$ PRR spectrum at different temperatures. The red and blue envelopes correspond to the temperature of 280 and $220 \mathrm{~K}$, respectively. The laser-beam wavelength is $354.67 \mathrm{~nm}$. The index over a spectral line denotes the rotational quantum number $J$ of the initial state of the transition. The spectral line number and number $J$ are the same for the Stokes branch. All PRR line intensities are normalized to the intensity of $\mathrm{N}_{2}$ PRR line with $J=6$ of the anti-Stokes branch at $T=220 \mathrm{~K}$.

al., 1983)

$$
\begin{aligned}
Q^{\Sigma}(T) & =\frac{I_{\text {low }}^{\Sigma}(T)}{I_{\text {high }}^{\Sigma}(T)} \\
& =\frac{\left[\sum_{J_{\mathrm{N}_{2}}} \beta_{\pi}\left(J_{\mathrm{N}_{2}}, T\right)+\sum_{J_{\mathrm{O}_{2}}} \beta_{\pi}\left(J_{\mathrm{O}_{2}}, T\right)\right]_{\text {low }}}{\left[\sum_{J_{\mathrm{N}_{2}}} \beta_{\pi}\left(J_{\mathrm{N}_{2}}, T\right)+\sum_{J_{\mathrm{O}_{2}}} \beta_{\pi}\left(J_{\mathrm{O}_{2}}, T\right)\right]_{\mathrm{high}}},
\end{aligned}
$$

where $\beta_{\pi}\left(J_{\mathrm{N}_{2}}, T\right)$ and $\beta_{\pi}\left(J_{\mathrm{O}_{2}}, T\right)$ are the backscatter coefficients corresponding to $\mathrm{N}_{2}$ and $\mathrm{O}_{2}$ individual PRR lines, respectively; $I_{\text {low }}^{\Sigma}(T)$ and $I_{\text {high }}^{\Sigma}(T)$ are the overall intensities of the PRR lines which enter the corresponding lidar temperature channels; indexes "low" and "high" show that summations in the numerator and denominator refer to the corresponding PRR-spectrum bands with $J_{\text {low }}$ and $J_{\text {high }}$. The ratio $Q^{\Sigma}(T)$ in Eq. (3) has a complicated temperature dependence and cannot be expressed as a simple function of $T$. For this reason, an approximation (calibration) function $f_{\mathrm{c}}^{\Sigma}(T)$ for the ratio $Q^{\Sigma}(T)$ is required to retrieve temperature profiles from lidar remote sensing data (Behrendt, 2005). The temperature retrieval accuracy and number of calibration coefficients depend on the selected calibration function.

Assuming that each PRR line profile represents the Dirac function, the general calibration function can be written in a natural logarithm form as follows (Gerasimov and Zuev, 
2016):

$$
\begin{aligned}
\ln Q^{\Sigma}(T) & \approx \ln f_{\mathrm{c}}^{\Sigma}(T)=A+\frac{B}{T}+\frac{C}{T^{2}}+\frac{D}{T^{3}} \\
& +\cdots \Leftrightarrow y=A+B x+C x^{2}+D x^{3}+\cdots,
\end{aligned}
$$

where $A, B, C, D$, etc. are the calibration (fit) coefficients determined by applying the least square method to lidar remote sensing (or simulation) data and reference radiosonde (or model) data; the symbol $\Leftrightarrow$ denotes the equivalence of expressions; $x=1 / T$ is the reciprocal temperature. The $n$ order in $x$ polynomial is assumed to retrieve temperature profiles with any desired accuracy depending on $n$ (Di Girolamo et al., 2004). The linear in $x$ special case of Eq. (4) with two calibration coefficients $A$ and $B$ (Arshinov et al., 1983) and the second-order in $x$ polynomial with three calibration coefficients $A, B$, and $C$ (Behrendt and Reichardt, 2000) are usually used by lidar researchers for temperature retrievals in the troposphere and lower stratosphere. However, $\mathrm{N}_{2}$ and $\mathrm{O}_{2}$ PRR lines are broadened by the Doppler and molecular collision effects. Hence, their backscatter profiles are described by a Voigt function, which is a convolution of certain Gaussian and Lorentzian functions (Nedeljkovic et al., 1993). As the molecular collision effect dominates over the Doppler effect in the troposphere (Ivanova et al., 1993), one can consider the Lorentzian function for a PRR line shape description instead of the Voigt one (Ginzburg, 1972). Therefore, all collisionally broadened PRR lines contribute to the signals detected in both lidar temperature channels due to the long Lorentzian tails of the line profiles (Measures, 1984), and the general calibration function takes on the form (Gerasimov and Zuev, 2016)

$$
\begin{aligned}
\ln Q^{\text {all }}(T) & =\cdots+\frac{A_{-2}}{T}+\frac{A_{-1}}{\sqrt{T}}+A_{0}+A_{1} \sqrt{T}+A_{2} T \\
& +\cdots=\sum_{n=-\infty}^{\infty} A_{n} T^{\frac{n}{2}}
\end{aligned}
$$

where $A_{n}$ are the calibration coefficients and Eq. (4) represents a special case of Eq. (5). All the calibration functions mentioned above are valid only when the parasitic elastic signal backscattered by atmospheric aerosols and molecules is sufficiently suppressed in the lidar temperature channels. The state-of-the-art narrow-band IFs and DGs provide the suppression of the parasitic signal intensity in the channels up to 8-10 orders of magnitude (Achtert et al., 2013; Hammann and Behrendt, 2015; Hammann et al., 2015).

In order to take into account the atmospheric extinction of backscattered signals and their losses in the lidar transmitting and receiving optics, one should consider the lidar equation (Measures, 1984)

$N(\lambda, z, T)=\eta N_{0} G(\lambda, z) \frac{c \tau_{0}}{2} \xi(\lambda) \frac{A}{z^{2}} \beta_{\pi}(\lambda, z, T) \Theta^{2}(\lambda, z)$,

where $N(\lambda, z, T)$ is the number of backscattered photons (photocounts) detected by a photomultiplier tube (PMT) in a lidar temperature channel, $N_{0}$ is the number of emitted photons, $\eta$ is the PMT quantum efficiency, $G(\lambda, z)$ is the laser-beam receiver-field-of-view overlap, $\tau_{0}$ is the laser pulse duration, $c$ is the speed of light, $\xi(\lambda)$ is the transmittance of the lidar receiving optical system, $A$ is the receiver telescope area, $z$ is the scattering region altitude, and $\Theta(\lambda, z)$ is the transmission coefficient through the atmosphere between the scattering region and the lidar. Taking Eqs. (5) and (6) into account, the ratio of the background-subtracted photocounts $N_{\mathrm{L}}$ and $N_{\mathrm{H}}$ from two spectrally close bands involving several $\mathrm{N}_{2}$ and $\mathrm{O}_{2}$ PRR lines with $J_{\text {low }}$ and $J_{\text {high }}$ becomes (Newsom et al., 2012; Newsom et al., 2013)

$$
\begin{aligned}
Q(T, z) & =\frac{N_{\mathrm{L}}(T, z)}{N_{\mathrm{H}}(T, z)}=\frac{G_{\mathrm{L}}(z)}{G_{\mathrm{H}}(z)} \exp \left(\sum_{n=-\infty}^{\infty} B_{n} T^{\frac{n}{2}}\right) \\
& =O(z) \exp \left(\sum_{n=-\infty}^{\infty} B_{n} T^{\frac{n}{2}}\right),
\end{aligned}
$$

where $B_{n}$ are the calibration coefficients and $O(z)=G_{\mathrm{L}}(z) / G_{\mathrm{H}}(z)$ is the laser-beam receiver-fieldof-view overlap function. At the complete overlap altitudes (usually above the atmospheric boundary layer), where $O(z)=1$, Eq. (7) goes over into the calibration function like Eq. (5):

$\ln Q(T)=\sum_{n=-\infty}^{\infty} B_{n} T^{\frac{n}{2}}$.

Note that the same result can be obtained on the assumption that the collisionally broadened elastic backscattered signal leaks into the nearest (to the laser line) lidar temperature channel (Gerasimov et al., 2015).

In our recent Optic Express paper, we considered the physics of our approach, derived mathematically the general calibration function that takes into account the collisional broadening of all $\mathrm{N}_{2}$ and $\mathrm{O}_{2}$ PRR lines, analyzed four nonlinear three-coefficient special cases of Eq. (8) via simulation to be used in the temperature retrieval algorithm, and determined the best function among them. In this paper, we apply these calibration functions to real lidar remote sensing data. The calibration function best suited for tropospheric temperature retrievals (for our PRR lidar system) is determined from the comparative analysis of temperature uncertainties yielded by using these functions.

\section{Special cases of the general calibration function}

The general calibration function expressed by Eq. (8) represents an infinite series and, hence, the temperature retrieval function $T=T(Q)$ cannot be obtained in an analytical form from this series. Therefore, one can use, for example, some special cases of the integer power approximation of Eq. (8), i.e.,

$\ln Q(T) \approx \cdots+\frac{C_{-2}}{T^{2}}+\frac{C_{-1}}{T}+C_{0}+C_{1} T+C_{2} T^{2}$ 


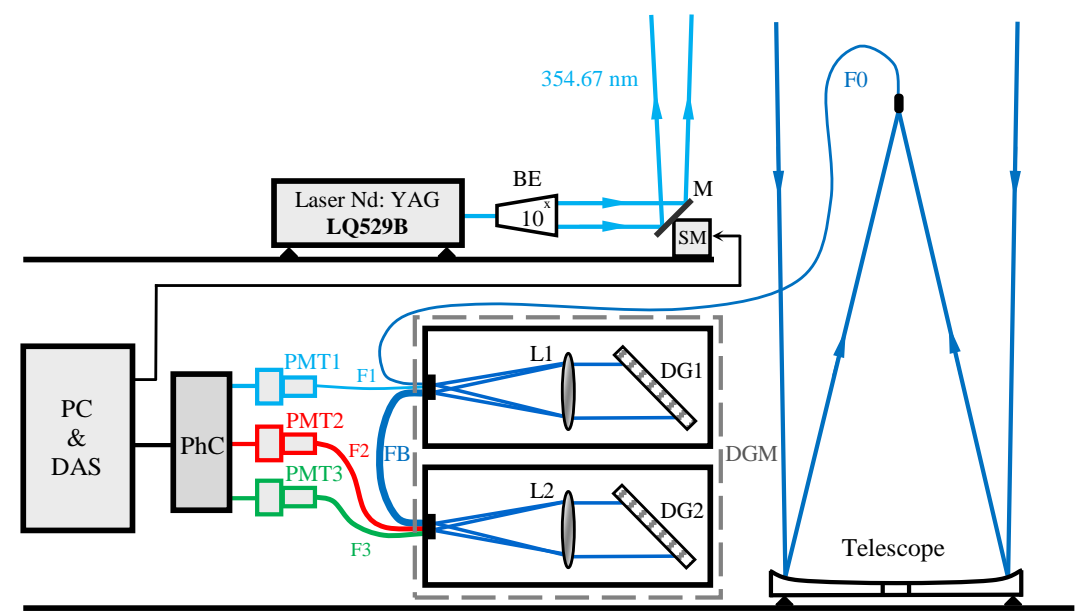

Figure 2. IMCES lidar optical layout (see also Table 1): PC \& DAS indicates personal computer and data acquisition system; PhC is the photon counter; PMT1-PMT3 are photomultiplier tubes; F0-F3 are optical fibers; FB is the four fiber bundle, connecting two monochromator blocks; DGM is the double-grating monochromator; L1 and L2 are lenses; DG1 and DG2 are diffraction gratings; BE is the beam expander with expansion factor of $10 ; \mathrm{M}$ is the mirror; $\mathrm{SM}$ is the stepping motor.
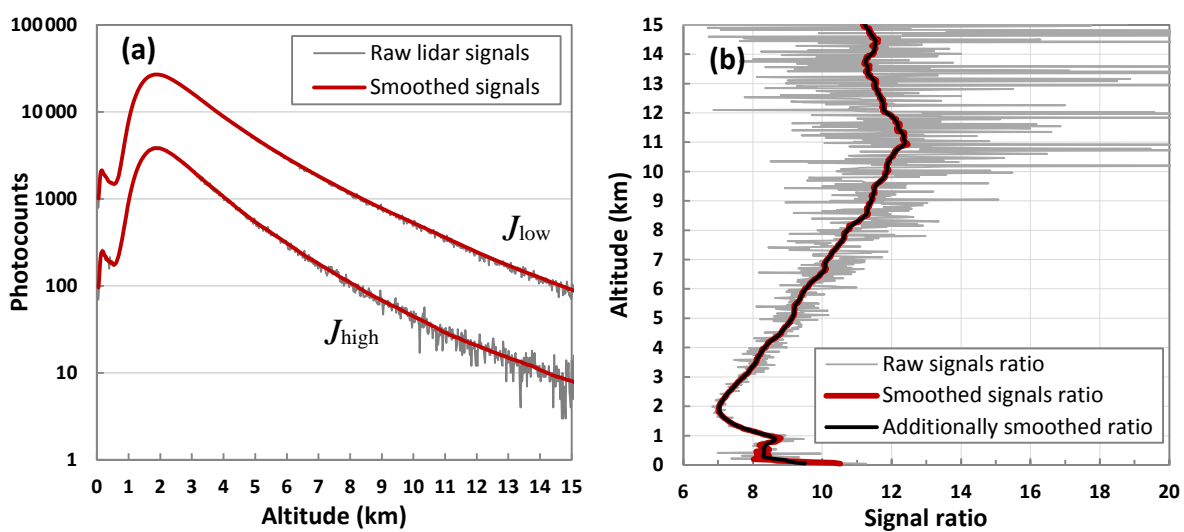

Figure 3. IMCES lidar data taken between 03:45 and 05:15 LT on 1 April 2015 (31 March, 21:45-23:15 UTC). (a) Raw photocounts $N_{\mathrm{L}}$ and $N_{\mathrm{H}}$ detected in the lidar channels with $J_{\text {low }}$ and $J_{\text {high }}$, respectively, together with the single-averaged ones $\bar{N}_{\mathrm{L}}$ and $\bar{N}_{\mathrm{H}}$. (b) Raw photocounts ratio $Q=N_{\mathrm{L}} / N_{\mathrm{H}}$, single-averaged photocounts ratio $Q=\bar{N}_{\mathrm{L}} / \bar{N}_{\mathrm{H}}$, and additionally averaged ratio $Q \mathrm{I}=\bar{Q}_{\mathrm{L}}=\overline{\bar{N}}_{\mathrm{L}} / \bar{N}_{\mathrm{H}}$.

$$
+\cdots=\sum_{n=-\infty}^{\infty} C_{n} T^{n}
$$

where $C_{n}$ are the calibration coefficients which can differ from $B_{n}$ in Eq. (8). Here we consider the linear and four simplest nonlinear (in reciprocal temperature $1 / T$ ) calibration functions and their corresponding temperature retrieval functions. Since Eq. (9) is a special case of Eq. (8), any special case of Eq. (9) automatically represents a special case of Eq. (8). The absolute and relative uncertainties of indirect temperature measurements are obtained in an analytical form in Appendices A, A1-A5.

The frequently used calibration function linear in $x=1 / T$ (Arshinov et al., 1983) is a special case of Eq. (9):

$\ln Q=A_{0}+\frac{B_{0}}{T} \Leftrightarrow y=A_{0}+B_{0} x$.
Its corresponding temperature retrieval function is

$$
T=\frac{B_{0}}{\ln Q-A_{0}},
$$

where $A_{0}$ and $B_{0}$ are the commonly designated calibration constants.

The most used nonlinear calibration function (Behrendt and Reichardt, 2000), containing the term quadratic in $x=1 / T$, also represents a special case of Eq. (9), i.e.,

$\ln Q=A_{1}+\frac{B_{1}}{T}+\frac{C_{1}}{T^{2}} \Leftrightarrow y=A_{1}+B_{1} x+C_{1} x^{2}$,

where $A_{1}, B_{1}$, and $C_{1}$ are the calibration constants. The corresponding temperature retrieval function is simply derived 


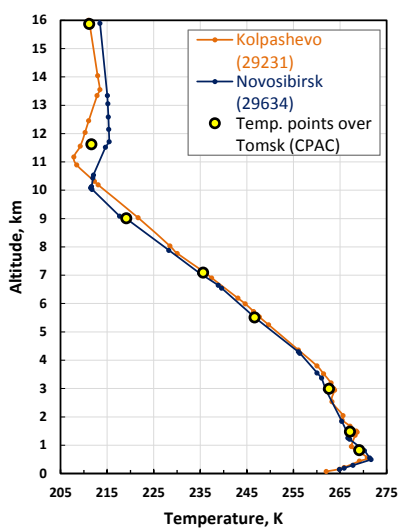

Figure 4. Temperature profiles from radiosondes launched on 1 April 2015 at 06:00 LT (00:00 UTC) in Novosibirsk (station 29634) and Kolpashevo (station 29231) as well as temperature points over Tomsk retrieved from constant pressure altitude charts (CPACs).

from Eq. (12)

$$
T=\frac{2 C_{1}}{-B_{1}+\sqrt{B_{1}^{2}+4 C_{1}\left(\ln Q-A_{1}\right)}} .
$$

Another three-coefficient special case of Eq. (9) can be written as follows (Gerasimov and Zuev, 2016):

$\ln Q=A_{2}+\frac{B_{2}}{T}+C_{2} T \Leftrightarrow y=A_{2}+B_{2} x+\frac{C_{2}}{x}$,

where $A_{2}, B_{2}$, and $C_{2}$ are the calibration constants. Solving Eq. (14), we have for the temperature retrieval function

$$
T=\frac{2 B_{2}}{\left(\ln Q-A_{2}\right)+\sqrt{\left(\ln Q-A_{2}\right)^{2}-4 B_{2} C_{2}}} .
$$

As it follows from the PRR lidar theory (Cooney, 1972), $y=\ln Q$ is a linear function of reciprocal temperature $x=1 / T$ (Arshinov et al., 1983). Conversely, the reciprocal temperature represents a linear function of $\ln Q$, i.e., $x=a+$ by. In order to take nonlinear effects into account, we consider the function

$x=a+b y+c y^{2} \Leftrightarrow \frac{1}{T}=a+b \ln Q+c(\ln Q)^{2}$,

where $a, b$, and $c$ are some constants. Thus, a temperature profile can simply be retrieved via

$T=\left[c(\ln Q)^{2}+b \ln Q+a\right]^{-1}$

or

$T=\frac{C_{3}}{(\ln Q)^{2}+B_{3} \ln Q+A_{3}}$,

where $A_{3}=a / c, B_{3}=b / c$, and $C_{3}=1 / c$. Equation (18) was first applied to real lidar data by Lee III (2013). Note that
Eq. (16) represents a special case of Eq. (8), as we showed in our 2016 paper.

There exists another way to represent collisional PRR lines broadening (and, therefore, nonlinear effects). Adding a term hyperbolic in $y=\ln Q$ to the linear calibration function of the form $x=a+b y$ gives

$x=A_{4}+B_{4} y+\frac{C_{4}}{y} \Leftrightarrow \frac{1}{T}=A_{4}+B_{4} \ln Q+\frac{C_{4}}{\ln Q}$,

where $A_{4}, B_{4}$, and $C_{4}$ are the calibration constants. Solving Eq. (19) yields

$$
\begin{aligned}
T & =\frac{1}{A_{4}+B_{4} \ln Q+\left(C_{4} / \ln Q\right)} \\
& =\frac{\ln Q}{B_{4}(\ln Q)^{2}+A_{4} \ln Q+C_{4}} .
\end{aligned}
$$

All the nonlinear calibration (or temperature retrieval) functions considered here take into account in varying degrees the collisional PRR lines broadening.

\section{The IMCES lidar setup}

The IMCES PRR lidar was developed in the Institute of Monitoring of Climatic and Ecological Systems of the Siberian Branch of the Russian Academy of Sciences (IMCES SB RAS) for nighttime tropospheric temperature measurements. A frequency-tripled Nd:YAG laser operating at a wavelength of $354.67 \mathrm{~nm}$ with $105 \mathrm{~mJ}$ pulse energy at a pulse repetition rate of $20 \mathrm{~Hz}$ is used as the lidar transmitter. The backscattered signals (photons) are collected by a prime-focus receiving telescope with a mirror diameter of $0.5 \mathrm{~m}$. The IMCES lidar optical layout is shown in Fig. 2. The selection of spectrum bands containing PRR lines with $J_{\text {low }}$ and $J_{\text {high }}$ from both the Stokes and anti-Stokes branches of $\mathrm{N}_{2}$ and $\mathrm{O}_{2}$ PRR spectra (Fig. 1) is performed via a double-grating monochromator (DGM). The DGM design and arrangement of optical fibers connecting both DGM blocks are the same as suggested by Ansmann et al. (1999). The main technical parameters of the IMCES lidar transmitting, receiving, and data acquisition systems are summarized in Table 1 . The spectral selection parameters of the DGM channels are listed in Table 2 .

\section{Temperature measurement example (1 April 2015)}

In this section we consider an example of nighttime tropospheric temperature measurements performed with the IMCES lidar on 1 April 2015 in Tomsk $\left(56.48^{\circ} \mathrm{N}, 85.05^{\circ} \mathrm{E}\right.$; Western Siberia, Russia). The lidar data were taken from 03:45 to 05:15 LT (or 31 March, 21:45-23:15 UTC), i.e., within 90 min integration time (108 000 laser shots). In order to determine the best calibration function that yields the minimum temperature retrieval uncertainties, we compare 

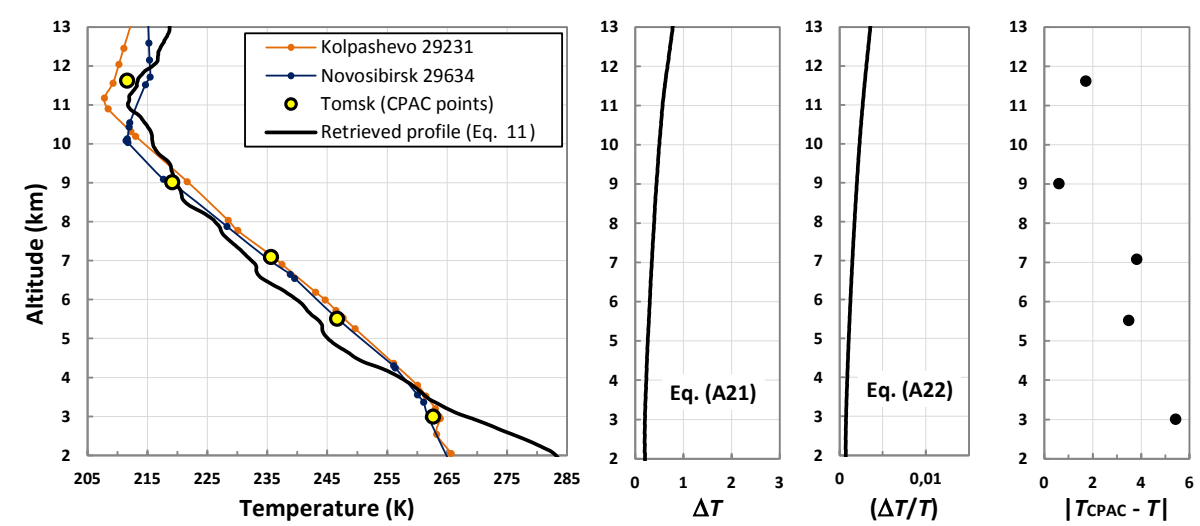

Figure 5. (1 April 2015) Temperature profile retrieved using the temperature retrieval function (Eq. 11) derived from the standard linear calibration function (Eq. 10, Arshinov et al., 1983). The absolute and relative uncertainties $\Delta T=\Delta \overline{\bar{T}}$ and $(\Delta T / T)=(\overline{\overline{\Delta T}} / T)$ are calculated by Eqs. (A21) and (A22), respectively. The values $T_{\mathrm{CPAC}}$ over Tomsk are retrieved from the 700, 500, 400, 300, and $200 \mathrm{hPa}$ constant pressure altitude charts (CPACs). The radiosonde data from the nearest station in Novosibirsk and Kolpashevo are given for comparison.
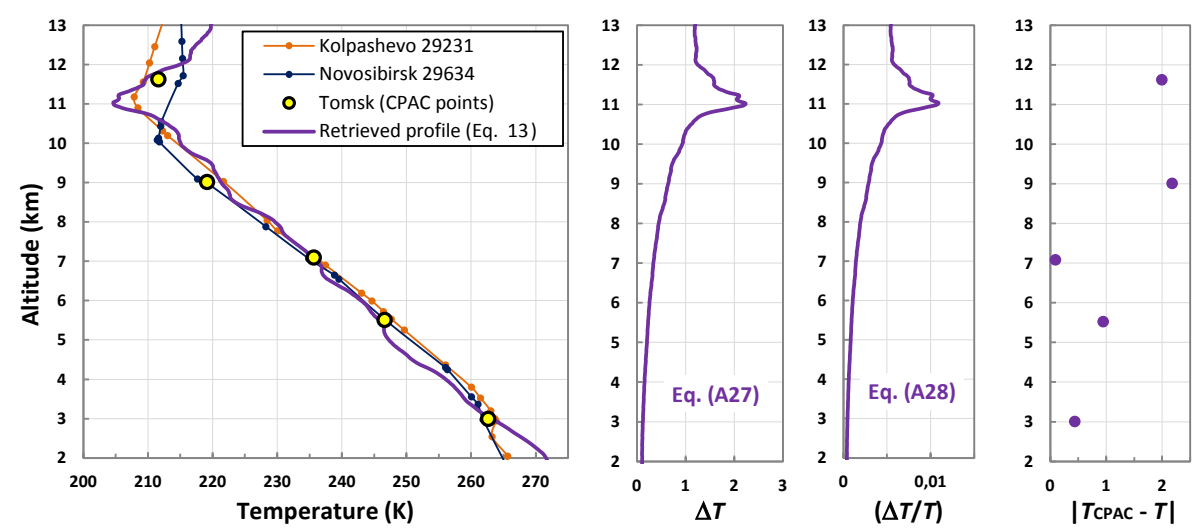

Figure 6. (1 April 2015) Temperature profile retrieved using the temperature retrieval function (Eq. 13) derived from the standard calibration function suggested by Behrendt and Reichardt (2000). The uncertainties $\Delta T$ and ( $\Delta T / T)$ are calculated by Eqs. (A27) and (A28), respectively.

and analyze five vertical tropospheric temperature profiles retrieved from the lidar data using Eqs. (11), (13), (15), (18), and (20).

\subsection{Raw lidar data averaging}

In order to improve the signal-to-noise ratio, raw lidar data (background-subtracted photocounts $N_{\mathrm{L}}$ and $N_{\mathrm{H}}$ detected by PMTs in the DGM channels) should be averaged. We tested more than dozens of different data-averaging methods including the equal-sized and variable sliding-window averaging ones presented in various papers (Behrendt and Reichardt, 2000; Behrendt et al., 2002; Alpers et al., 2004; Di Girolamo et al., 2004; Radlach et al., 2008; Radlach, 2009; Jia and Yi, 2014). The optimal data-averaging method for our lidar system is the following. The IMCES lidar raw data with vertical resolution of $\Delta z=24 \mathrm{~m}$ are averaged with a variable sliding average window (Appendix A). Having an initial size of $n=2 k+1=3(k=1)$, the sliding window is increased by one point on either side of the central point for every 10 data points. Otherwise, starting with an initial length of $\Delta \bar{z}=n \Delta z=72 \mathrm{~m}$ in the lidar to $240 \mathrm{~m}$ altitude range, the sliding window is increased above and below by $24 \mathrm{~m}$ for every $240 \mathrm{~m}$ increase in altitude (see Fig. 3a). For example, the sliding window size and length (or averaged data resolution) are of $n=27(k=13)$ and $\Delta \bar{z}=648 \mathrm{~m}$ at an altitude of $3 \mathrm{~km}$ and $n=85(k=42)$ and $\Delta \bar{z}=2040 \mathrm{~m}$ at an altitude of $10 \mathrm{~km}$, respectively. Note that similar lidar-data-averaging procedure was used, e.g., in Lee III (2013). Due to low power of the IMCES lidar laser, the ratio of single-averaged signals (i.e., $Q=\bar{N}_{\mathrm{L}} / \bar{N}_{\mathrm{H}}$ ) was additionally slightly averaged with a small equal-sized sliding window $(l=5$, and $m=11$ in Eq. A7) to reduce signal statistical fluctuations (Fig. 3b; see also the Supplement). For example, the double-averaged data resolution becomes $\Delta \overline{\bar{z}}=[2(k+l)+1] \Delta z=2280 \mathrm{~m}(k=42$, $l=5)$ at an altitude of $10 \mathrm{~km}$, but both absolute and relative statistical uncertainties additionally decrease by $\sqrt{m}=\sqrt{11}$ 

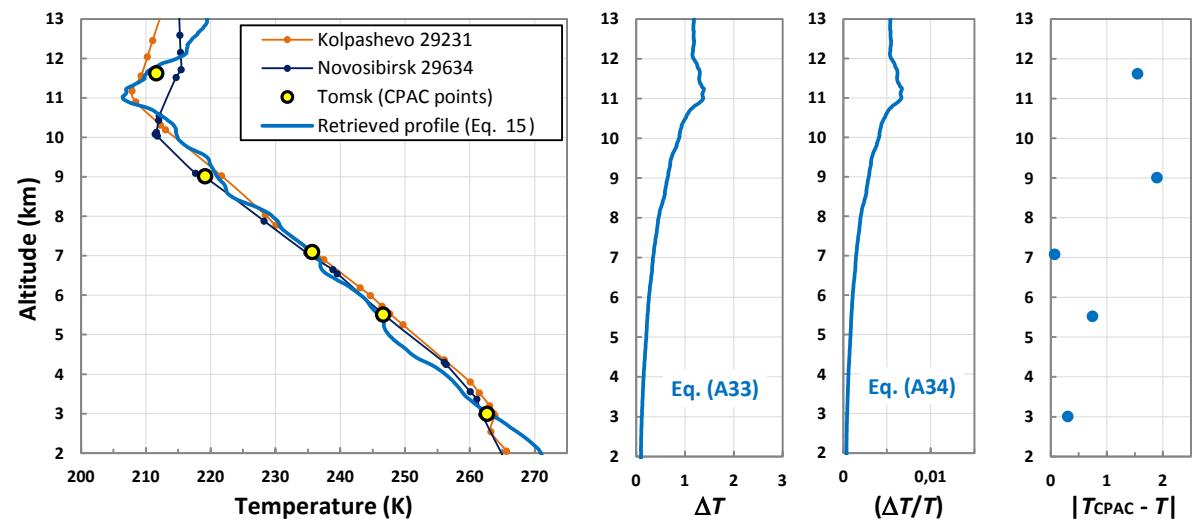

Figure 7. (1 April 2015) Temperature profile retrieved using the temperature retrieval function (Eq. 15) derived from the calibration function suggested by Gerasimov and Zuev (2016). The uncertainties $\Delta T$ and ( $\Delta T / T)$ are calculated by Eqs. (A33) and (A34), respectively.
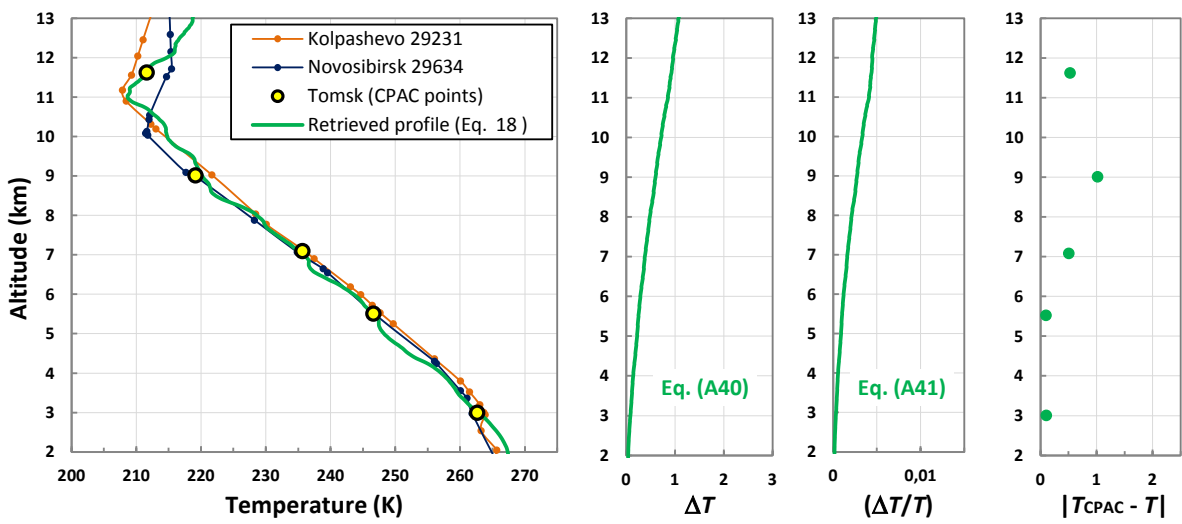

Figure 8. (1 April 2015) Temperature profile retrieved using the temperature retrieval function (Eq. 18) derived from the calibration function suggested by Lee III (2013). The uncertainties $\Delta T$ and $(\Delta T / T)$ are calculated by Eqs. (A40) and (A41), respectively.

times (Appendix A). For any other lidar system, the optimal data-averaging method can differ from the method we used.

\subsection{Reference temperature points for the lidar calibration}

One of the problems we face during temperature measurements is as follows. Unfortunately, we do not have our own radiosondes and, therefore, we have no possibility to launch a radiosonde simultaneously with lidar remote sensing at the lidar site. The two nearest to Tomsk meteorological stations launching radiosondes twice a day are situated in Novosibirsk $\left(55.02^{\circ} \mathrm{N}, 82.92^{\circ} \mathrm{E}\right)$ and Kolpashevo $\left(58.32^{\circ} \mathrm{N}\right.$, $82.92^{\circ} \mathrm{E}$ ). Both towns are at a distance of more than $250 \mathrm{~km}$ from Tomsk. Hence, we cannot directly use vertical temperature profiles from these radiosondes as reference data points, which are known to be required for PRR lidars calibration. However, we solved this problem by using temperature and altitude data from the 925, 850, 700, 500, 400, 300, 200 , and $100 \mathrm{hPa}$ constant pressure altitude charts (CPACs) as "reference" data to obtain several points over Tomsk for the IMCES lidar calibration. Six CPACs are presented in the Supplement as an example. Original CPACs can be found at http://gpu.math.tsu.ru/maps/. Assuming the uncertainty to be half of the least significant digit, the required points were determined by linear interpolation (Saucier, 2003) with the temperature accuracy of $0.5 \mathrm{~K}$ and the vertical accuracy of $5 \mathrm{~m}$. It is clear that the CPAC points are not quite suitable for use as the reference points to calibrate lidars and retrieve temperature profiles with high accuracy (for this purpose the local radiosonde data are required). Nevertheless, the accuracy of these points $(0.5 \mathrm{~K}, 5 \mathrm{~m})$ is sufficient to make the comparative analysis of temperature uncertainties, yielded by using different calibration functions, and determine the best-suited function (among them) for our lidar system. Two temperature profiles from radiosondes, launched on 1 April 2015 at 06:00 LT (00:00 UTC) in Novosibirsk and Kolpashevo, together with temperature points over Tomsk retrieved from the CPACs are shown in Figs. 4-9. The radiosonde data are presented only for comparison and can be found on the web page http://weather.uwyo.edu/upperair/sounding.html? region=np of the University of Wyoming (Novosibirsk and 

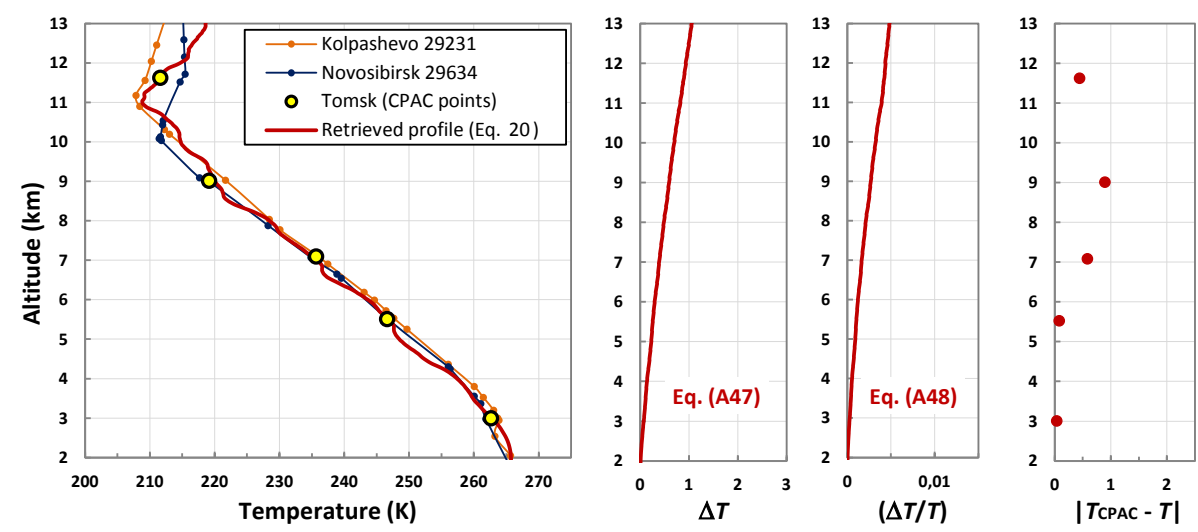

Figure 9. (1 April 2015) Temperature profile retrieved using the temperature retrieval function (Eq. 20) derived from the calibration function suggested by Gerasimov and Zuev (2016). The uncertainties $\Delta T$ and ( $\Delta T / T$ ) are calculated by Eqs. (A47) and (A48), respectively.

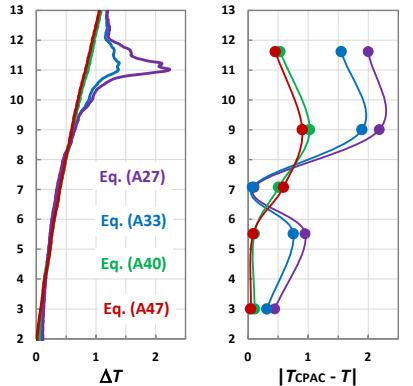

Figure 10. (1 April 2015) Comparative analysis of the absolute temperature uncertainties yielded by using Eqs. (A27), (A33), (A40), and (A47) and of the difference in modulus between temperature values retrieved from the CPACs and IMCES lidar data.

Kolpashevo station numbers are 29634 and 29231, respectively).

\subsection{Temperature profiles retrieved with different calibration functions}

Here we compare nighttime temperature profiles retrieved using five calibration functions considered in Sect. 2 from the altitude where the laser-beam receiver-field-of-view overlap is complete $(\sim 3 \mathrm{~km})$ to $13 \mathrm{~km}$ (i.e., slightly above the local tropopause). Figure 5 presents a tropospheric temperature profile retrieved using the temperature retrieval function (Eq. 11) derived from the standard linear calibration function (Eq. 10). The absolute statistical uncertainty $\Delta \overline{\bar{T}}$ of temperature retrieval is calculated by Eq. (A21), whereas the relative uncertainty $(\overline{\overline{\Delta T}} / T)$ is calculated by Eq. (A22). The difference in modulus $\left|T_{\mathrm{CPAC}}-T\right|$ between temperature values retrieved from the CPACs and IMCES lidar data is also presented in Fig. 5. The nearest radiosonde data are given for comparison. Figures 6-9 show temperature profiles retrieved using the temperature retrieval functions expressed by Eqs. (13), (15), (18), and (20), respectively. These func- tions are derived from the corresponding nonlinear calibration functions, i.e., Eqs. (12), (14), (16), and (19).

Comparing all five profiles among themselves, one can see that, despite the lowest values of both the statistical uncertainties in the $3-12 \mathrm{~km}$ altitude region $(\Delta \overline{\bar{T}}<0.7 \mathrm{~K}$, $(\overline{\overline{\Delta T}} / T)<0.004)$ yielded by using Eq. (11), the difference $\left|T_{\text {CPAC }}-T\right|$ can reach $\sim 5.5 \mathrm{~K}$ (Fig. 5). For the nonlinear functions in the same altitude region, the maximum difference $\left|T_{\mathrm{CPAC}}-T\right|$ is less than 2.2 and $\sim 0.9 \mathrm{~K}$ when using Eq. (13) and Eq. (20), respectively, as seen in Figs. 6, 9, and 10 (see also the Supplement). Similarly, for both the uncertainties we have $\Delta \overline{\bar{T}}<2.3 \mathrm{~K},(\overline{\overline{\Delta T}} / T)<0.011$ when applying Eq. (13), and $\Delta \overline{\bar{T}}<1 \mathrm{~K},(\overline{\overline{\Delta T}} / T)<0.005$ for Eq. (20). Note that the peaks of curves $\Delta \overline{\bar{T}}$ and $(\overline{\overline{\Delta T}} / T)$ near $11 \mathrm{~km}$ altitude in Figs. 6 and 7 are caused by the problem with square roots in Eqs. (13) and (15) described in Appendices $\mathrm{A} 2$ and $\mathrm{A} 3$. There is no such problem in the case of Eqs. (18) and (20) without square roots. The tropopause is also located near $11 \mathrm{~km}$ altitude. Taking into account all three parameters $\Delta \overline{\bar{T}},(\overline{\overline{\Delta T}} / T)$, and $\left|T_{\mathrm{CPAC}}-T\right|$, we can conclude that Eqs. (13), (15), (18), and (20) retrieve the tropospheric temperature much better compared to Eq. (11). Moreover, the functions expressed by Eqs. (18) and (20) yield the smallest uncertainties and $\left|T_{\mathrm{CPAC}}-T\right|$ values among considered nonlinear functions and, therefore, they are the best suited for tropospheric temperature retrievals with the IMCES PRR lidar.

\section{Temperature measurement example (2 October 2014)}

Let us consider another example of nighttime tropospheric temperature measurements performed with the IMCES PRR lidar on 2 October 2014 in Tomsk. The lidar data were taken from 20:21 to 21:21 LT (13:21-14:21 UTC), i.e., within $60 \mathrm{~min}$ integration time (72000 laser shots). The raw and averaged IMCES lidar signals together with raw and aver- 
Table 1. Main technical parameters of the IMCES lidar transmitting, receiving, and data acquisition systems.

\begin{tabular}{|c|c|}
\hline \multicolumn{2}{|c|}{ Transmitting system } \\
\hline \multicolumn{2}{|l|}{ Laser } \\
\hline Type & Unseeded frequency-tripled Nd:YAG \\
\hline Model & Solar LS LQ529B \\
\hline Wavelength & $354.67 \mathrm{~nm}$ \\
\hline Spectral line width & $\sim 1 \mathrm{~cm}^{-1}$ \\
\hline Pulse repetition rate & $20 \mathrm{~Hz}$ \\
\hline Pulse energy & $105 \mathrm{~mJ}$ \\
\hline Pulse duration & $13 \mathrm{~ns}$ \\
\hline Beam divergence & $0.3 \mathrm{mrad}$ \\
\hline Expansion factor & 10 \\
\hline \multicolumn{2}{|c|}{ Receiving system } \\
\hline \multicolumn{2}{|l|}{ Telescope } \\
\hline Type & Prime-focus \\
\hline Receiving mirror diameter & $0.5 \mathrm{~m}$ \\
\hline Focal length & $1.5 \mathrm{~m}$ \\
\hline Field of view & $0.4 \mathrm{mrad}$ \\
\hline \multicolumn{2}{|l|}{ Optical fibers } \\
\hline F0 input fiber diameter & $0.55 \mathrm{~mm}$ (FG 550 UER) \\
\hline F1 output fiber diameter & $0.6 \mathrm{~mm}(\mathrm{FT} 600 \mathrm{UMT})$ \\
\hline FB intermediate fibers diameter & $0.6 \mathrm{~mm}(\mathrm{FT} 600 \mathrm{UMT})$ \\
\hline F2 and F3 output fibers diameter & $1.5 \mathrm{~mm}(\mathrm{FT} 1.5 \mathrm{UMT})$ \\
\hline \multicolumn{2}{|c|}{ Double-grating monochromator } \\
\hline \multicolumn{2}{|l|}{ Lens L1, L2 } \\
\hline Diameter & $130 \mathrm{~mm}$ \\
\hline Focal length & $300 \mathrm{~mm}$ \\
\hline \multicolumn{2}{|l|}{ Diffraction gratings DG1, DG2 } \\
\hline Grooves $\mathrm{mm}^{-1}$ & 2100 \\
\hline Diffraction order & 2 \\
\hline Diffraction angle & $48.151^{\circ}$ \\
\hline \multicolumn{2}{|c|}{ Data acquisition system } \\
\hline Photomultiplier tubes PMT1-PMT3 & Hamamatsu R7207-01 \\
\hline PMTs quantum efficiency & $25 \%$ \\
\hline Photon counter & PHCOUNT_4 (IMCES SB RAS) \\
\hline Number of channels & 4 ( 3 in use) \\
\hline Counting rate & Up to 200 counts $s^{-1}$ \\
\hline Initial vertical resolution & $24 \mathrm{~m}$ \\
\hline
\end{tabular}

aged signal ratios are presented in Fig. 11. Here also we compare five temperature profiles retrieved using Eqs. (11), (13), (15), (18), and (20). The temperature retrieval algorithm is the same as was applied to the IMCES lidar data dated 1 April 2015. For the lidar calibration, we retrieved temperature points over Tomsk using the corresponding CPACs. Two temperature profiles from radiosondes, launched on 2 October 2014 at 19:00 LT (12:00 UTC) in Novosibirsk and Kolpashevo, are also given for comparison.
Figure 12 shows a temperature profile retrieved using Eq. (11). For this profile in the $3-12 \mathrm{~km}$ altitude region we have $\Delta \overline{\bar{T}}<1 \mathrm{~K},(\overline{\overline{\Delta T}} / T)<0.005$, and $\left|T_{\mathrm{CPAC}}-T\right|<$ $6.5 \mathrm{~K}$. Figure 13 shows temperature profiles retrieved using Eqs. (13) and (18). The temperature profiles retrieved using Eqs. (15) and (20) are presented in Fig. 14. As seen, e.g., in Fig. 14, $\Delta \overline{\bar{T}}<1.8 \mathrm{~K},(\overline{\overline{\Delta T}} / T)<0.009$, and $\left|T_{\mathrm{CPAC}}-T\right|<2.9 \mathrm{~K}$ when applying Eq. (15); and 

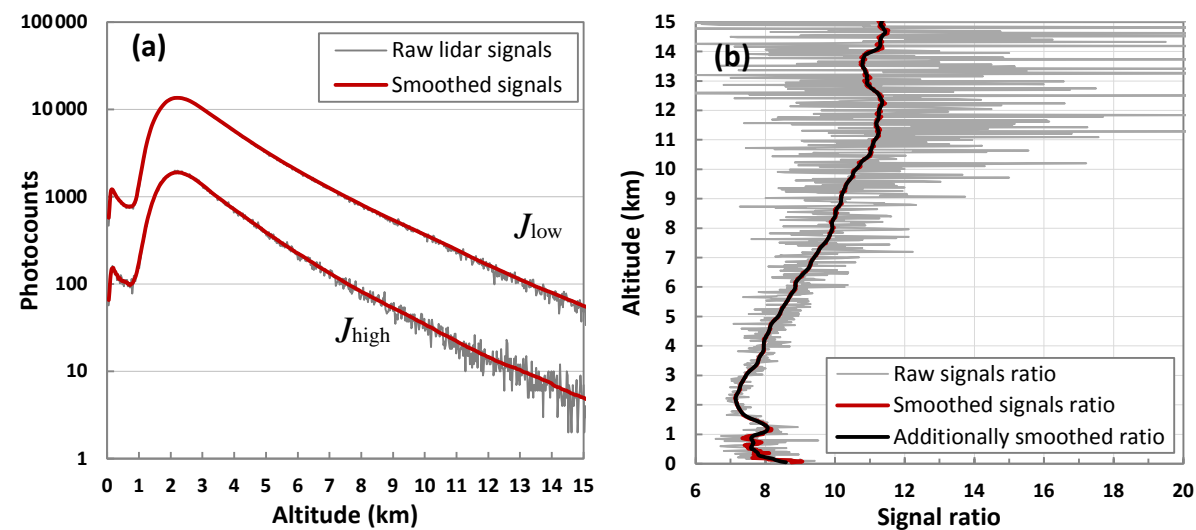

Figure 11. IMCES lidar data taken between 20:21 and 21:21 LT on 2 October 2014 (13:21-14:21 UTC). (a) Raw photocounts $N_{\mathrm{L}}$ and $N_{\mathrm{H}}$ detected in the lidar channels with $J_{\text {low }}$ and $J_{\text {high }}$, respectively, together with the single-averaged ones $\bar{N}_{\mathrm{L}}$ and $\bar{N}_{\mathrm{H}}$. (b) Raw photocounts ratio $Q=N_{\mathrm{L}} / N_{\mathrm{H}}$, single-averaged photocounts ratio $Q=\bar{N}_{\mathrm{L}} / \bar{N}_{\mathrm{H}}$, and additionally averaged ratio $Q_{\mathrm{I}}=\bar{Q}_{=} \overline{\bar{N}}_{\mathrm{L}} / \bar{N}_{\mathrm{H}}$.
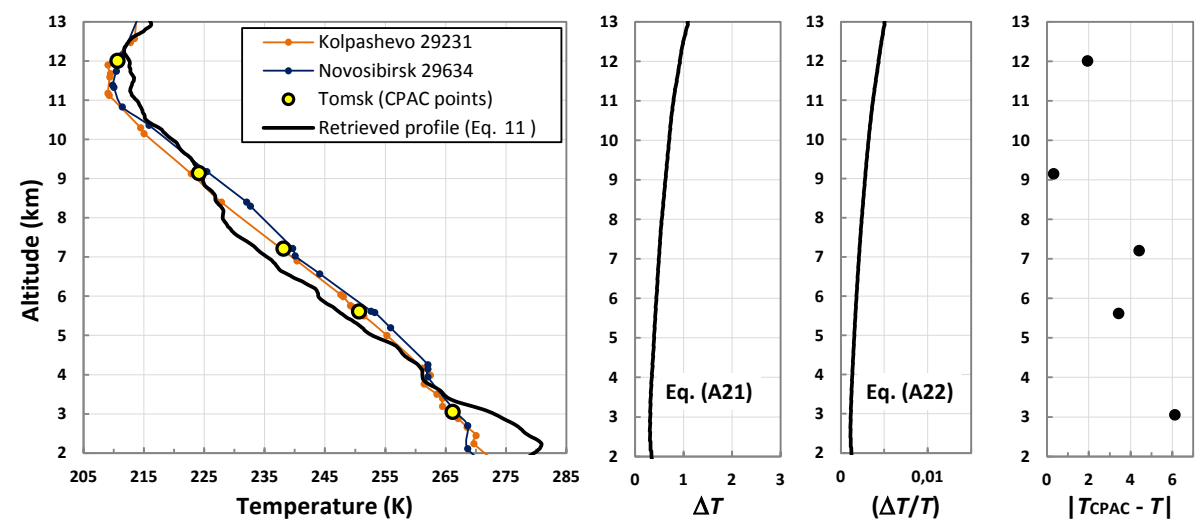

Figure 12. (2 October 2014) Temperature profile retrieved using Eq. (11). The absolute and relative uncertainties $\Delta T=\Delta \overline{\bar{T}}$ and $(\Delta T / T)=$ $(\overline{\overline{\Delta T}} / T)$ are calculated by Eqs. (A21) and (A22), respectively.

Table 2. Spectral selection parameters of the DGM channels (central wavelength (CWL) and full width at half maximum (FWHM)).

\begin{tabular}{lll}
\hline DGM channel & CWL $(\mathrm{nm})$ & FWHM $\left(\mathrm{nm} / \mathrm{cm}^{-1}\right)$ \\
$J_{\text {low }}($ Stokes $)$ & 355.22 & $\sim 0.22 / 17$ \\
$J_{\text {low }}$ (anti-Stokes) & 354.12 & $\sim 0.22 / 17$ \\
$J_{\text {high }}($ Stokes $)$ & 356.03 & $\sim 0.35 / 28$ \\
$J_{\text {high }}$ (anti-Stokes) & 353.32 & $\sim 0.35 / 28$ \\
\hline
\end{tabular}

$\Delta \overline{\bar{T}}<1.3 \mathrm{~K},(\overline{\overline{\Delta T}} / T)<0.007$, and $\left|T_{\mathrm{CPAC}}-T\right|<1.8 \mathrm{~K}$ for Eq. (20) in the $3-12 \mathrm{~km}$ altitude region. The comparative analysis of the parameters is presented in Fig. 15. The tropopause is located near $12.3 \mathrm{~km}$ altitude. Comparing pairwise all the retrieved profiles for both measurement examples, one can see that $\Delta \overline{\bar{T}},(\overline{\overline{\Delta T}} / T)$, and $\left|T_{\text {CPAC }}-T\right|$ values in case of the second example (2 October 2014) are higher than that for the first one (1 April 2015, Sect. 4.3). This is due to the smaller number of laser shots (and, therefore, photo- counts detected in both DGM channels) leading to the higher absolute and relative statistical uncertainties, as seen from Eqs. (A9) and (A10) in Appendix A. The two best-suited functions for temperature retrievals are seen in Figs. 13 and 14 to be the same as in the previous example (1 April 2015). The large difference between the CPAC and lidar temperature values in 2 to $3 \mathrm{~km}$ altitude region (Figs. 5 and 12; see also Lee III, 2013) is, perhaps, due to the incomplete laser-beam receiver-field-of-view overlap in the region. We also cannot exclude that any of the nonlinear calibration functions are able to somehow correct for this incomplete overlap in the atmospheric boundary layer.

The calibration coefficients of all the calibration functions used in both the temperature measurement examples can be found in the Supplement. 

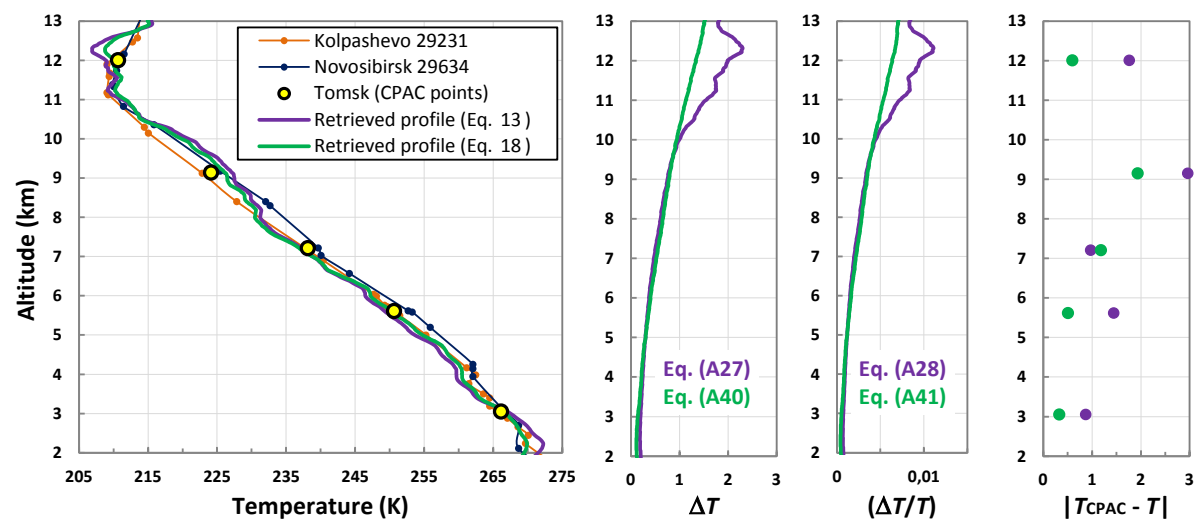

Figure 13. (2 October 2014) Temperature profiles retrieved using Eqs. (13) and (18).
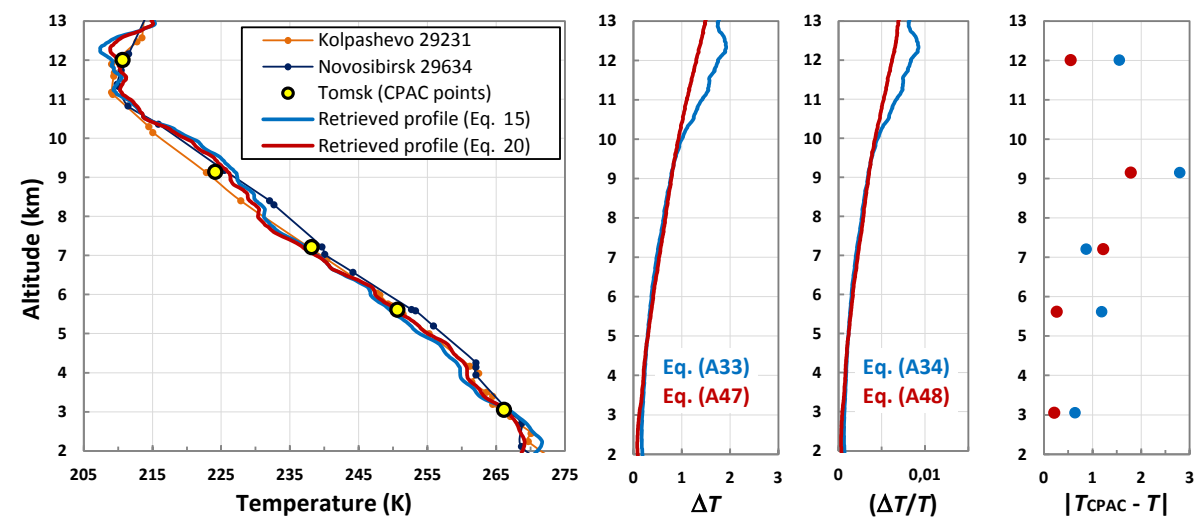

Figure 14. (2 October 2014) Temperature profiles retrieved using Eqs. (15) and (20).

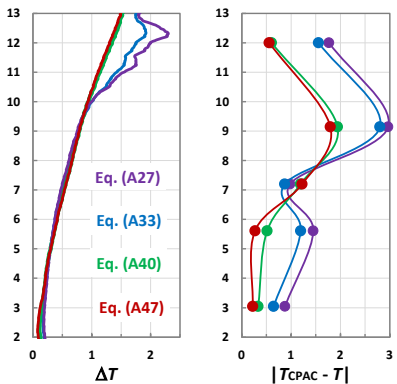

Figure 15. (2 October 2014) Comparative analysis of the absolute temperature uncertainties yielded by using Eqs. (A27), (A33), (A40), and (A47) and of the difference in modulus between temperature values retrieved from the CPACs and IMCES lidar data.

\section{Summary and outlook}

We have considered and used the linear and four nonlinear (three-coefficient) in $x=1 / T$ calibration functions in the tropospheric temperature retrieval algorithm. The corresponding temperature retrieval functions were applied to the nighttime temperature measurement data obtained with the IMCES PRR lidar on 2 October 2014 and 1 April 2015. We have also derived and used the absolute and relative statistical uncertainties of indirect temperature measurements in an analytical form (Appendices A, A1-A5).

For the case of the IMCES PRR lidar system, the comparative analysis of three parameters $\Delta \overline{\bar{T}},(\overline{\overline{\Delta T}} / T)$, and $\left|T_{\mathrm{CPAC}}-T\right|$ showed the following:

- the nonlinear functions expressed by Eqs. (13), (15), (18), and (20) retrieve the tropospheric temperature much better compared to the linear function (Eq. 11);

- Eqs. (18) and (20) give the almost equally best-suited functions for the tropospheric temperature retrievals (although Eq. (20) is slightly better than Eq. 18);

- the function given by Eq. (18) is the best from both practical (real lidar data) and theoretical (simulation) points of view (Gerasimov and Zuev, 2016).

As it was mentioned previously (Sect. 4.2), the CPAC points can hardly be used as the reference data to reliably calibrate PRR lidars and retrieve accurate temperature profiles. Nevertheless, the results suggest that the best-suited calibration function for temperature retrievals can depend on the lidar system (e.g., based on DGs or IFs for PRR lines extracting), 
which can take into account the collisional broadening of PRR lines in varying degrees. Indeed, the calibration errors depend on the spectral characteristics of the lidar receiver such as the central wavelength, shape and width of the transmission functions, and whether just the anti-Stokes (IFs) or both branches of the PRR spectrum (DGs) are used to extract the PRR signals from backscattered light. Therefore, it is reasonable to check all the mentioned nonlinear functions against lidar data obtained with different lidar systems to determine the best function in each specific case. Furthermore, the stability of the calibration functions coefficients during long-time lidar measurements is one of the crucial aspects in determination of the best function. Hence, it would be a good thing to study the coefficients stability during a night (Jia and Yi, 2014; Li et al., 2015), week, month, etc. as it was done in Lee III (2013) for the linear calibration function coefficients.

\section{Data availability}

The radiosonde data are available on the web page http:// weather.uwyo.edu/upperair/sounding.html?region=np of the University of Wyoming. Original CPACs can be found at http://gpu.math.tsu.ru/maps/. The IMCES PRR lidar raw and averaged signals (signal ratios) together with the processed CPACs are presented in the Supplement. 


\section{Appendix A: Absolute and relative uncertainties of temperature retrieval}

Each value $T$ of a temperature profile retrieved from raw lidar data is known to be within the confidence interval $[T-\Delta T$; $T+\Delta T$ ], where $\Delta T>0$. Assuming Poisson statistics of photon counting, the $1-\sigma$ absolute statistical uncertainty $\Delta T$ of indirect temperature measurements is defined in the general form as follows (Behrendt, 2005; Radlach, 2009):

$$
\Delta T=\sqrt{\left(\frac{\mathrm{d} T}{\mathrm{~d} Q} \Delta Q\right)^{2}}=\left|\frac{\mathrm{d} T}{\mathrm{~d} Q}\right| Q \sqrt{\frac{1}{N_{\mathrm{H}}}+\frac{1}{N_{\mathrm{L}}}},
$$

where the temperature retrieval function $T=T(Q)$ is derived from any required calibration function (see Sect. 2); $Q=N_{\mathrm{L}} / N_{\mathrm{H}}$ is the ratio of the background-subtracted photocounts $N_{\mathrm{L}}$ and $N_{\mathrm{H}}$ registered in the lidar temperature channels with $J_{\text {low }}$ and $J_{\text {high }}$, respectively. Consequently, the relative statistical uncertainty $(\Delta T / T)$ of indirect temperature measurements is simply derived from Eq. (A1):

$$
\left(\frac{\Delta T}{T}\right)=\left|\frac{\mathrm{d} T}{\mathrm{~d} Q}\right| \frac{Q}{T} \sqrt{\frac{1}{N_{\mathrm{H}}}+\frac{1}{N_{\mathrm{L}}}} .
$$

However, Eqs. (A1) and (A2) are valid only for unaveraged (raw) lidar data $N_{\mathrm{L}}$ and $N_{\mathrm{H}}$. In practice, raw data are previously averaged to improve the signal-to-noise ratio. One of the most simple and used data-averaging methods is the equal-sized (or variable) sliding-window averaging (Behrendt and Reichardt, 2000; Behrendt et al., 2002; Alpers et al., 2004; Di Girolamo et al., 2004; Radlach et al., 2008; Radlach, 2009; Lee III, 2013). The averaged data $\bar{N}(z)$ and their variance $\overline{\operatorname{Var}}(z)$ are related to the corresponding unaveraged data $N(z)$ and variance $\operatorname{Var}(z)$ as follows (El'nikov et al., 2000):

$$
\begin{aligned}
\bar{N}_{j}(z) & =\frac{1}{2 k+1} \sum_{i=-k}^{k} N_{j+i}=\frac{1}{2 k+1} \sum_{i=-k}^{k} N(z+i \Delta z) \\
& =\frac{1}{n}[N(z-k \Delta z)+\cdots+N(z)+\cdots+N(z+k \Delta z)],
\end{aligned}
$$

$$
\overline{\operatorname{Var}}(z)=\operatorname{Var}(z) / n,
$$

where $\Delta z$ is the vertical resolution of raw lidar data (initial vertical resolution); $k$ is the number of data points on either side of the central point $N_{j}$; and $n=2 k+1$ is the sliding average window size, i.e., the number of raw lidar data points determining the sliding average window length or data resolution after averaging (Otnes and Enochson, 1978). The weighting coefficients of the raw data points in Eq. (A3) are the same and equal to $1 /(2 k+1)$. The vertical resolution of the averaged data series $\left\{\overline{N_{j}}\right\}$ is $\Delta \bar{z}=n \Delta z=(2 k+1) \Delta z$. As the variance decreases by $n$ times, the absolute uncertainty $\Delta \bar{N}(z)$ of averaged data decreases by $\sqrt{n}$ times. Therefore, for the absolute uncertainty of temperature retrieval from the averaged lidar data (photocounts) $\bar{N}_{\mathrm{H}}$ and $\bar{N}_{\mathrm{L}}$ we have

$$
\Delta \bar{T}=\frac{\Delta T}{\sqrt{n}}=\left|\frac{\mathrm{d} T}{\mathrm{~d} Q}\right| \frac{Q}{\sqrt{n}} \sqrt{\frac{1}{\bar{N}_{\mathrm{H}}}+\frac{1}{\bar{N}_{\mathrm{L}}},}
$$

where $Q=\bar{N}_{\mathrm{L}} / \bar{N}_{\mathrm{H}}$. Hence, the confidence interval of the retrieved temperature profile is $[T-\Delta \bar{T} ; T+\Delta \bar{T}]$, and the relative uncertainty is given by

$\left(\overline{\frac{\Delta T}{T}}\right)=\left|\frac{\mathrm{d} T}{\mathrm{~d} Q}\right| \frac{Q}{T \sqrt{n}} \sqrt{\frac{1}{\bar{N}_{\mathrm{H}}}+\frac{1}{\bar{N}_{\mathrm{L}}}}$.

In some cases, the second-order averaging of raw data (or/and their ratio) is required and more preferable than the first-order one (see, e.g., El'nikov et al., 2000). In such cases, the double-averaged data $\overline{\bar{N}}(z)$ and their variance $\overline{\overline{\operatorname{Var}}}(z)$ are related to the corresponding single-averaged data $\bar{N}(z)$, and variances $\overline{\operatorname{Var}}(z)$ and $\operatorname{Var}(z)$ as follows:

$\overline{\bar{N}}_{j}(z)=\frac{1}{2 l+1} \sum_{i=-l}^{l} \bar{N}_{j+i}=\frac{1}{m} \sum_{i=-l}^{l} \bar{N}(z+i \Delta z)$,

$\overline{\overline{\operatorname{Var}}}(z)=\overline{\operatorname{Var}}(z) / m=\operatorname{Var}(z) /(n m)$,

where $l$ is the number of the single-averaged data points on either side of the central point $\bar{N}_{j}$ and $m=2 l+1$ is the sliding average window size. The confidence interval of a retrieved temperature profile is $[T-\Delta \overline{\bar{T}} ; T+\Delta \overline{\bar{T}}]$, where $\Delta \overline{\bar{T}}=\Delta T / \sqrt{n m}$.

There are two ways to average previously averaged PRR lidar data. The first way is to average the ratio $Q=\bar{N}_{\mathrm{L}} / \bar{N}_{\mathrm{H}}$ of the single-averaged data $\bar{N}_{\mathrm{H}}$ and $\bar{N}_{\mathrm{L}}$. In this case, the absolute and relative uncertainties of temperature retrieval from the averaged ratio $Q_{\mathrm{I}}=\bar{Q}=\overline{\bar{N}}_{\mathrm{L}} / \bar{N}_{\mathrm{H}}$ are given by

$$
\begin{aligned}
& \Delta \overline{\bar{T}}=\left|\frac{\mathrm{d} T}{\mathrm{~d} Q_{\mathrm{I}}}\right| \frac{Q_{\mathrm{I}}}{\sqrt{n m}} \sqrt{\frac{1}{\bar{N}_{\mathrm{H}}}+\frac{1}{\bar{N}_{\mathrm{L}}},} \\
& \left(\frac{\overline{\overline{\Delta T}}}{T}\right)=\left|\frac{\mathrm{d} T}{\mathrm{~d} Q_{\mathrm{I}}}\right| \frac{Q_{\mathrm{I}}}{T \sqrt{n m}} \sqrt{\frac{1}{\bar{N}_{\mathrm{H}}}+\frac{1}{\bar{N}_{\mathrm{L}}}} .
\end{aligned}
$$

The second way is to average the single-averaged data $\bar{N}_{\mathrm{H}}$ and $\bar{N}_{\mathrm{L}}$. The absolute and relative uncertainties of temperature retrieval from the double-averaged lidar data $\overline{\bar{N}}_{\mathrm{H}}$ and $\overline{\bar{N}}_{\mathrm{L}}$ (and for $Q_{\mathrm{II}}=\overline{\bar{N}}_{\mathrm{L}} / \overline{\bar{N}}_{\mathrm{H}}$ ) are determined by

$$
\begin{aligned}
& \Delta \overline{\bar{T}}=\left|\frac{\mathrm{d} T}{\mathrm{~d} Q_{\mathrm{II}}}\right| \frac{Q_{\mathrm{II}}}{\sqrt{n m}} \sqrt{\frac{1}{\overline{\bar{N}}_{\mathrm{H}}}+\frac{1}{\overline{\bar{N}}_{\mathrm{L}}},} \\
& \left(\frac{\overline{\overline{\Delta T}}}{T}\right)=\left|\frac{\mathrm{d} T}{\mathrm{~d} Q_{\mathrm{II}}}\right| \frac{Q_{\mathrm{II}}}{T \sqrt{n m}} \sqrt{\frac{1}{\overline{\bar{N}}_{\mathrm{H}}}+\frac{1}{\overline{\bar{N}}_{\mathrm{L}}}} .
\end{aligned}
$$


The vertical resolution of the double-averaged data series $\left\{\overline{\bar{N}}_{j}\right\}$ and for both ways of the second-order averaging becomes

$\Delta \overline{\bar{z}}=p \Delta z=[2(k+l)+1] \Delta z$.

If the window size $n$ (and/or $m$ ) varies with altitude $z$, both the uncertainties should be estimated separately for each altitude interval where $n=$ const (and/or $m=$ const). To determine the weighting coefficients of the raw data points in Eq. (A7), it is necessary to consider three possible simple cases of the second-order averaging.

1. Let $k>l(n>m)$; i.e., the sliding average window size for the first-order averaging is larger than that for the second-order one. Then

$$
\begin{aligned}
\overline{\bar{N}}_{j} & =\frac{1}{(2 l+1)(2 k+1)} \\
& \left\{(2 l+1)\left[N_{j}+\sum_{i=1}^{k-l}\left(N_{j-i}+N_{j+i}\right)\right]\right. \\
& \left.+\sum_{i=k-l+1}^{k+l}(k+l+1-i)\left(N_{j-i}+N_{j+i}\right)\right\} .
\end{aligned}
$$

The weighting coefficients can be determined from Eq. (A14) of the following form:

$$
\begin{aligned}
\overline{\bar{N}}_{j} & =\frac{1}{2 k+1}\left[N_{j}+\sum_{i=1}^{k-l}\left(N_{j-i}+N_{j+i}\right)\right] \\
& +\sum_{i=k-l+1}^{k+l} \frac{k+l+1-i}{(2 l+1)(2 k+1)}\left(N_{j-i}+N_{j+i}\right) .
\end{aligned}
$$

2. Let $l>k(m>n)$; i.e., the window size for the secondorder averaging is larger than that for the first-order one. Then

$$
\begin{aligned}
\overline{\bar{N}}_{j} & =\frac{1}{(2 k+1)(2 l+1)}\{(2 k+1) \\
& {\left[N_{j}+\sum_{i=1}^{l-k}\left(N_{j-i}+N_{j+i}\right)\right] } \\
& \left.+\sum_{i=l-k+1}^{l+k}(l+k+1-i)\left(N_{j-i}+N_{j+i}\right)\right\} .
\end{aligned}
$$

The corresponding weighting coefficients are determined similar to case (1).

3. Let $l=k(m=n)$; i.e., the window size for the secondorder averaging is equal to that for the first-order one. Then

$$
\overline{\bar{N}}_{j}=\frac{1}{(2 k+1)^{2}}
$$

$$
\left\{(2 k+1) N_{j}+\sum_{i=1}^{2 k}(2 k+1-i)\left(N_{j-i}+N_{j+i}\right)\right\} .
$$

The weighting coefficients are determined similar to cases (1) and (2). The vertical resolution of the doubleaveraged data series $\left\{\overline{\bar{N}}_{j}\right\}$ in case (3) is $\Delta \overline{\bar{z}}=p \Delta z=$ $(4 k+1) \Delta z$ (El'nikov et al., 2000).

\section{A1 Linear calibration function}

As we applied the first way of the second-order averaging of the IMCES lidar raw data (see Appendix A and Sect. 4.1), we use Eqs. (A9) and (A10) to derive the absolute and relative uncertainties in an analytical form. In case of the first-order averaging of lidar raw data, one can use Eqs. (A5) and (A6), respectively.

In order to obtain both the uncertainties for the linear calibration function, let us differentiate the temperature retrieval function derived from Eq. (10), i.e. (see Sect. 2)

$T=\frac{B_{0}}{\ln Q-A_{0}}$.

The first-order derivative of the function is

$\frac{\mathrm{d} T}{\mathrm{~d} Q}=-\frac{B_{0}}{Q\left(\ln Q-A_{0}\right)^{2}}$.

Substituting Eq. (A19) into Eq. (A9), for the absolute uncertainty we get

$\Delta \overline{\bar{T}}=\frac{\left|B_{0}\right|}{\left(\ln Q_{\mathrm{I}}-A_{0}\right)^{2} \sqrt{n m}} \sqrt{\frac{1}{\bar{N}_{\mathrm{H}}}+\frac{1}{\bar{N}_{\mathrm{L}}} .}$

One can rewrite Eq. (A20) in more simple form by substituting the expression $\ln Q-A_{0}=B_{0} / T$ derived from Eq. (A18)

$$
\Delta \overline{\bar{T}}=\frac{T^{2}}{\left|B_{0}\right| \sqrt{n m}} \sqrt{\frac{1}{\bar{N}_{\mathrm{H}}}+\frac{1}{\bar{N}_{\mathrm{L}}}} .
$$

Consequently, substituting Eq. (A19) into Eq. (A10), for the relative uncertainty we have

$$
\begin{aligned}
\left(\overline{\overline{\Delta T}} \frac{1}{T}\right) & =\frac{1}{\left|\ln Q_{\mathrm{I}}-A_{0}\right| \sqrt{n m}} \sqrt{\frac{1}{\bar{N}_{\mathrm{H}}}+\frac{1}{\bar{N}_{\mathrm{L}}}} \\
& =\frac{T}{\left|B_{0}\right| \sqrt{n m}} \sqrt{\frac{1}{\bar{N}_{\mathrm{H}}}+\frac{1}{\bar{N}_{\mathrm{L}}}} .
\end{aligned}
$$

\section{A2 Calibration function quadratic in $x=1 / T$}

The temperature retrieval function derived from Eq. (12) is written as (see Sect. 2)

$$
T=\frac{2 C_{1}}{-B_{1} \pm \sqrt{B_{1}^{2}+4 C_{1}\left(\ln Q-A_{1}\right)}}
$$


The sign "+" instead of " \pm " should be chosen in the denominator of Eq. (A23), if $Q=N_{\mathrm{L}} / N_{\mathrm{H}}$. When applying Eq. (A23) for temperature retrievals, one should take into account the constraint coming from the square root. Namely, the expression under the square root should be nonnegative, i.e., $B_{1}^{2}+$ $4 C_{1}\left[\ln Q(z)-A_{1}\right] \geq 0$ or $\ln Q(z) \leq\left(B_{1}^{2} / 4 C_{1}\right)-A_{1}$. Hence, Eq. (A23) can retrieve the temperature profile $T$ only at altitudes $z$ where this condition holds.

The first-order derivative of the function is

$$
\frac{\mathrm{d} T}{\mathrm{~d} Q}=\frac{-4 C_{1}^{2}\left[-B_{1}+\sqrt{B_{1}^{2}+4 C_{1}\left(\ln Q-A_{1}\right)}\right]^{-2}}{Q \sqrt{B_{1}^{2}+4 C_{1}\left(\ln Q-A_{1}\right)}} .
$$

It is clear that the expressions for both absolute and relative uncertainties will be cumbersome and poorly adapted for use after substitution of this derivative in Eqs. (A9) and (A10). However, Eq. (A24) can be put in a more convenient form by substituting the expressions which follow from Eq. (A23):

$$
\begin{aligned}
-B_{1}+\sqrt{B_{1}^{2}+4 C_{1}\left(\ln Q-A_{1}\right)} & =\frac{2 C_{1}}{T}, \\
\sqrt{B_{1}^{2}+4 C_{1}\left(\ln Q-A_{1}\right)} & =\frac{2 C_{1}}{T}+B_{1} .
\end{aligned}
$$

After substitution of Eqs. (A25) into Eq. (A24), we can write instead of Eq. (A24)

$$
\frac{\mathrm{d} T}{\mathrm{~d} Q}=\frac{-T^{3}}{Q\left(2 C_{1}+B_{1} T\right)} .
$$

Substituting Eq. (A26) into Eqs. (A9) and (A10), we obtain correspondingly for the absolute and relative uncertainties

$$
\begin{aligned}
& \Delta \overline{\bar{T}}=\frac{T^{3}}{\left|2 C_{1}+B_{1} T\right| \sqrt{n m}} \sqrt{\frac{1}{\bar{N}_{\mathrm{H}}}+\frac{1}{\bar{N}_{\mathrm{L}}},} \\
& (\overline{\overline{\Delta T}})=\frac{T^{2}}{\left|2 C_{1}+B_{1} T\right| \sqrt{n m}} \sqrt{\frac{1}{\bar{N}_{\mathrm{H}}}+\frac{1}{\bar{N}_{\mathrm{L}}}} .
\end{aligned}
$$

\section{A3 Calibration function hyperbolic in $x=1 / T$}

The temperature retrieval function in the general form derived from Eq. (14) represents (see Sect. 2)

$$
T=\frac{2 B_{2}}{\left(\ln Q-A_{2}\right) \pm \sqrt{\left(\ln Q-A_{2}\right)^{2}-4 B_{2} C_{2}}} .
$$

For the case of $Q=N_{\mathrm{L}} / N_{\mathrm{H}}$, the sign "+" instead of " \pm " should also be chosen in the denominator of Eq. (A29). Note that Eq. (A29) can retrieve the temperature $T$ only at altitudes $z$ where the following condition holds: $\left[\ln Q(z)-A_{2}\right]^{2}-$ $4 B_{2} C_{2} \geq 0$ or $\ln Q(z) \geq A_{2}+2 \sqrt{B_{2} C_{2}}$ (with $B_{2} C_{2} \geq 0$ ).

The derivative of the temperature retrieval function is

$$
\frac{\mathrm{d} T}{\mathrm{~d} Q}=\frac{2 B_{2}}{Q\left[\left(\ln Q-A_{2}\right)+\sqrt{\left(\ln Q-A_{2}\right)^{2}-4 B_{2} C_{2}}\right]^{2}}
$$

$$
\times\left[1+\frac{\ln Q-A_{2}}{\sqrt{\left(\ln Q-A_{2}\right)^{2}-4 B_{2} C_{2}}}\right] .
$$

Equation (A30) can be put in a more convenient form by substituting the expressions which follow from Eqs. (A29) and (14), respectively

$$
\begin{gathered}
\left(\ln Q-A_{2}\right)+\sqrt{\left(\ln Q-A_{2}\right)^{2}-4 B_{2} C_{2}}=2 B_{2} / T, \\
\ln Q-A_{2}=B_{2} / T+C_{2} T .
\end{gathered}
$$

After substitution of Eqs. (A31) into Eq. (A30), we get for the derivative

$\frac{\mathrm{d} T}{\mathrm{~d} Q}=\frac{T^{2}}{Q\left(B_{2}-C_{2} T^{2}\right)}$.

Then substituting Eq. (A32) into Eqs. (A9) and (A10), we obtain for both the uncertainties

$$
\begin{aligned}
& \Delta \overline{\bar{T}}=\frac{T^{2}}{\left|B_{2}-C_{2} T^{2}\right| \sqrt{n m}} \sqrt{\frac{1}{\overline{\bar{N}}_{\mathrm{H}}}+\frac{1}{\overline{\bar{N}}_{\mathrm{L}}}}, \\
& \left(\frac{\overline{\overline{\Delta T}}}{T}\right)=\frac{T}{\left|B_{2}-C_{2} T^{2}\right| \sqrt{n m}} \sqrt{\frac{1}{\bar{N}_{\mathrm{H}}}+\frac{1}{\bar{N}_{\mathrm{L}}}} .
\end{aligned}
$$

\section{A4 Calibration function quadratic in $y=\ln Q$}

The first-order derivative of the temperature retrieval function, obtained from Eq. (16) (see Sect. 2)

$T=\frac{C_{3}}{(\ln Q)^{2}+B_{3} \ln Q+A_{3}}$,

is simply expressed as

$$
\frac{\mathrm{d} T}{\mathrm{~d} Q}=\frac{-C_{3}\left(2 \ln Q+B_{3}\right)}{Q\left[(\ln Q)^{2}+B_{3} \ln Q+A_{3}\right]^{2}} .
$$

Substituting Eq. (A36) into Eq. (A9), for the absolute uncertainty we get

$\Delta \overline{\bar{T}}=\frac{\left|C_{3}\left(2 \ln Q_{\mathrm{I}}+B_{3}\right)\right|}{\left[\left(\ln Q_{\mathrm{I}}\right)^{2}+B_{3} \ln Q_{\mathrm{I}}+A_{3}\right]^{2} \sqrt{n m}} \sqrt{\frac{1}{\bar{N}_{\mathrm{H}}}+\frac{1}{\bar{N}_{\mathrm{L}}}}$.

Using the expression derived from Eq. (A35), i.e.,

$(\ln Q)^{2}+B_{3} \ln Q+A_{3}=C_{3} / T$,

for the relative uncertainty we obtain

$(\overline{\overline{\Delta T}} \bar{T})=\left|\frac{2 \ln Q_{\mathrm{I}}+B_{3}}{\left(\ln Q_{\mathrm{I}}\right)^{2}+B_{3} \ln Q_{\mathrm{I}}+A_{3}}\right| \frac{1}{\sqrt{n m}} \sqrt{\frac{1}{\bar{N}_{\mathrm{H}}}+\frac{1}{\bar{N}_{\mathrm{L}}}}$.

In order to estimate both the uncertainties, one can also use Eqs. (A37) and (A39) in a more simple form. Substituting Eq. (A38) in Eqs. (A37) and (A39), we obtain the following equations containing both $\ln Q_{I}$ and retrieved temperature $T$ :

$$
\begin{aligned}
& \Delta \overline{\bar{T}}=\left|\frac{2 \ln Q_{\mathrm{I}}+B_{3}}{C_{3}}\right| \frac{T^{2}}{\sqrt{n m}} \sqrt{\frac{1}{\bar{N}_{\mathrm{H}}}+\frac{1}{\bar{N}_{\mathrm{L}}},} \\
& \left(\frac{\overline{\overline{\Delta T}}}{T}\right)=\left|\frac{2 \ln Q_{\mathrm{I}}+B_{3}}{C_{3}}\right| \frac{T}{\sqrt{n m}} \sqrt{\frac{1}{\bar{N}_{\mathrm{H}}}+\frac{1}{\bar{N}_{\mathrm{L}}}} .
\end{aligned}
$$




\section{A5 Calibration function hyperbolic in $y=\ln Q$}

Tropospheric temperature profiles are mentioned in Sect. 2 can also be retrieved via the function

$T=\frac{\ln Q}{B_{4}(\ln Q)^{2}+A_{4} \ln Q+C_{4}}$,

the first-order derivative of which is defined as

$\frac{\mathrm{d} T}{\mathrm{~d} Q}=\frac{C_{4}-B_{4}(\ln Q)^{2}}{Q\left[B_{4}(\ln Q)^{2}+A_{4} \ln Q+C_{4}\right]^{2}}$.

Substituting Eq. (A43) in Eq. (A9), we obtain the absolute uncertainty containing only $\ln Q$ :

$\Delta \overline{\bar{T}}=\frac{\left|C_{4}-B_{4}\left(\ln Q_{\mathrm{I}}\right)^{2}\right|}{\left[B_{4}\left(\ln Q_{\mathrm{I}}\right)^{2}+A_{4} \ln Q_{\mathrm{I}}+C_{4}\right]^{2} \sqrt{n m}} \sqrt{\frac{1}{\bar{N}_{\mathrm{H}}}+\frac{1}{\bar{N}_{\mathrm{L}}}}$.

Using the expression derived from Eq. (A42), i.e.,

$B_{4}(\ln Q)^{2}+A_{4} \ln Q+C_{4}=(\ln Q) / T$,

for the relative uncertainty we get

$$
\begin{aligned}
(\overline{\overline{\Delta T}} \bar{T})= & \left|\frac{C_{4}-B_{4}\left(\ln Q_{\mathrm{I}}\right)^{2}}{B_{4}\left(\ln Q_{\mathrm{I}}\right)^{3}+A_{4}\left(\ln Q_{\mathrm{I}}\right)^{2}+C_{4} \ln Q_{\mathrm{I}}}\right| \\
& \frac{1}{\sqrt{n m}} \sqrt{\frac{1}{\bar{N}_{\mathrm{H}}}+\frac{1}{\bar{N}_{\mathrm{L}}}} .
\end{aligned}
$$

Similarly, using Eq. (A45), one can rewrite Eqs. (A44) and (A46) in a practically useful form:

$$
\begin{aligned}
& \Delta \overline{\bar{T}}=\left|\frac{C_{4}}{\left(\ln Q_{\mathrm{I}}\right)^{2}}-B_{4}\right| \frac{T^{2}}{\sqrt{n m}} \sqrt{\frac{1}{\bar{N}_{\mathrm{H}}}+\frac{1}{\bar{N}_{\mathrm{L}}}}, \\
& \left(\frac{\overline{\overline{\Delta T}}}{T}\right)=\left|\frac{C_{4}}{\left(\ln Q_{\mathrm{I}}\right)^{2}}-B_{4}\right| \frac{T}{\sqrt{n m}} \sqrt{\frac{1}{\bar{N}_{\mathrm{H}}}+\frac{1}{\bar{N}_{\mathrm{L}}}} .
\end{aligned}
$$




\section{The Supplement related to this article is available online at doi:10.5194/amt-10-315-2017-supplement.}

Acknowledgements. We thank S. M. Bobrovnikov for helpful discussions. This study was conducted in the framework of the Federal Targeted Programme "R\&D in Priority Fields of S\&T Complex of Russia for 2014-2020" in the priority field "Rational use of natural resources" (contract no. 14.607.21.0030, unique identifier ASR RFMEFI60714X0030)

Edited by: R. Sica

Reviewed by: A. Hauchecorne and one anonymous referee

\section{References}

Achtert, P., Khaplanov, M., Khosrawi, F., and Gumbel, J.: Pure rotational-Raman channels of the Esrange lidar for temperature and particle extinction measurements in the troposphere and lower stratosphere, Atmos. Meas. Tech., 6, 91-98, doi:10.5194/amt-6-91-2013, 2013.

Alpers, M., Eixmann, R., Fricke-Begemann, C., Gerding, M., and Höffner, J.: Temperature lidar measurements from 1 to $105 \mathrm{~km}$ altitude using resonance, Rayleigh, and Rotational Raman scattering, Atmos. Chem. Phys., 4, 793-800, doi:10.5194/acp-4-7932004, 2004.

Ansmann, A., Arshinov Y., Bobrovnikov, S. M., Mattis, I., Serikov, I. B., and Wandinger, U.: Double-grating monochromator for a pure rotational Raman lidar, Proc. SPIE, 3583, 491-497, doi:10.1117/12.337058, 1999.

Arshinov, Y. F., Bobrovnikov, S. M., Zuev, V. E., and Mitev, V. M.: Atmospheric temperature measurements using a pure rotational Raman lidar, Appl. Optics, 22, 2984-2990, 1983.

Behrendt, A.: Temperature measurements with lidar, in: Lidar: Range-Resolved Optical Remote Sensing of the Atmosphere, edited by: Weitkamp, C., Springer, New York, 273-305, 2005.

Behrendt A. and Reichardt, J.: Atmospheric temperature profiling in the presence of clouds with a pure rotational Raman lidar by use of an interference-filter-based polychromator, Appl. Optics, 39, 1372-1378, 2000.

Behrendt, A., Nakamura, T., Onishi, M., Baumgart, R., and Tsuda, T.: Combined Raman lidar for the measurement of atmospheric temperature, water vapor, particle extinction coefficient, and particle backscatter coefficient, Appl. Optics, 41, 7657-7666, 2002.

Behrendt, A., Wulfmeyer, V., Hammann, E., Muppa, S. K., and Pal, S.: Profiles of second- to fourth-order moments of turbulent temperature fluctuations in the convective boundary layer: first measurements with rotational Raman lidar, Atmos. Chem. Phys., 15, 5485-5500, doi:10.5194/acp-15-5485-2015, 2015.

Chen, S., Qiu, Z., Zhang, Y., Chen, H., and Wang, Y.: A pure rotational Raman lidar using double-grating monochromator for temperature profile detection, J. Quant. Spectr. Rad. Transfer, 112, 304-309, 2011.

Cooney, J.: Measurement of atmospheric temperature profiles by Raman backscatter, J. Appl. Meteorol., 11, 108-112, 1972.
Di Girolamo, P., Marchese, R., Whiteman, D. N., and Demoz, B. B.: Rotational Raman Lidar measurements of atmospheric temperature in the UV, Geophys. Res. Lett., 31, L01106, doi:10.1029/2003GL018342, 2004.

El'nikov, A. V., Zuev, V. V., and Bondarenko, S. L.: Retrieving the profiles of stratospheric ozone from lidar sensing data, Atmos. Ocean. Optics, 13, 1029-1034, 2000.

Gerasimov, V. V. and Zuev, V. V.: Analytical calibration functions for the pure rotational Raman lidar technique, Opt. Express, 24, 5136-5151, 2016.

Gerasimov, V. V., Zuev, V. V., Pravdin, V. L., Nakhtigalova, D. P., and Pavlinskiy, A. V.: Collision broadening effect upon tropospheric temperature calibration functions for pure rotational Raman lidars, Proc. SPIE, 9680, 96804F, doi:10.1117/12.2205832, 2015.

Ginzburg, V. L.: Line width in the spectrum of scattered light, Sov. Phys. Usp., 15, 114-120, 1972.

Hammann, E., Behrendt, A., Le Mounier, F., and Wulfmeyer, V.: Temperature profiling of the atmospheric boundary layer with rotational Raman lidar during the $\mathrm{HD}(\mathrm{CP}) 2$ Observational Prototype Experiment, Atmos. Chem. Phys., 15, 2867-2881, doi:10.5194/acp-15-2867-2015, 2015.

Ivanova, I. D., Gurdev, L. L., and Mitev, V. M.: Lidar technique for simultaneous temperature and pressure measurement based on rotation Raman scattering, J. Mod. Opt., 40, 367-371, 1993.

Jia, J. and Yi, F.: Atmospheric temperature measurements at altitudes of 5-30 km with a double-grating-based pure rotational Raman lidar, Appl. Optics, 53, 5330-5343, 2014.

Kim, D., Cha, H., Lee, J., and Bobronikov, S.: Pure rotational Raman lidar for atmospheric temperature measurements, J. Korean Phys. Soc., 39, 838-841, 2001.

Lee III, R. B.: Tropospheric temperature measurements using a rotational Raman lidar, Ph.D. dissertation, Hampton University, Hampton, Virginia, 112 pp., 2013.

Li, Y.-J., Song, S.-L., Li, F.-Q., Cheng X.-W., Chen, Z.-W., Liu, L.M., Yang, Y., and Gong, S.-S.: High-precision measurements of lower atmospheric temperature based on pure rotational Raman lidar, Chinese J. Geophys., 58, 2294-2305, 2015.

Measures, R. M.: Laser remote sensing. Fundamentals and applications, John Wiley \& Sons, New York, 94-102 and 237-243, 1984.

Nedeljkovic, D., Hauchecorne, A., and Chanin, M.-L.: Rotational Raman lidar to measure the atmospheric temperature from ground to $30 \mathrm{~km}$, IEEE T. Geosci. Remote, 31, 90-101, 1993.

Newsom, R. K., Sivaraman, C., and McFarlane, S. A.: Raman lidar profiles-temperature (RLPROFTEMP) value-added product: available at: https://www.arm.gov/publications/tech_reports/ doe-sc-arm-tr-120.pdf (last access: 24 January 2017), 2012.

Newsom, R. K., Turner, D. D., and Goldsmith, J. E. M.: Long-term evaluation of temperature profiles measured by an operational Raman lidar, J. Atmos. Ocean. Tech., 30, 1616-1634, 2013.

Otnes, R. K. and Enochson, L.: Applied Time Series Analysis. Vol. 1. Basic Techniques, John Wiley \& Sons, New York, 106-218, 1978.

Penney, C. M., Peters St., R. L., and Lapp, M.: Absolute rotational Raman cross sections for $\mathrm{N}_{2}, \mathrm{O}_{2}$, and $\mathrm{CO}_{2}$, J. Opt. Soc. Am., 64, 712-716, 1974

Radlach, M.: A scanning eye-safe rotational Raman lidar in the ultraviolet for measurements of tropospheric temperature fields, 
Ph.D. dissertation, University of Hohenheim, Stuttgart, Germany, 117 pp., 2009.

Radlach, M., Behrendt, A., and Wulfmeyer, V.: Scanning rotational Raman lidar at $355 \mathrm{~nm}$ for the measurement of tropospheric temperature fields, Atmos. Chem. Phys., 8, 159-169, doi:10.5194/acp-8-159-2008, 2008.

Saucier, W. J.: Theory and practice of scalar analysis, in: Principles of Meteorological Analysis, Dover Phoenix Edition, Dover Publications, Mineola, NY, 96-133, 2003.
Wandinger, U.: Raman lidar, in: Lidar: Range-Resolved Optical Remote Sensing of the Atmosphere, edited by: Weitkamp, C., Springer, New York, 241-271, 2005.

Wulfmeyer, V., Hardesty, R. M., Turner, D. D., Behrendt, A., Cadeddu, M. P., Di Girolamo, P., Schlüssel, P., Van Baelen, J., and Zus, F.: A review of the remote sensing of lower tropospheric thermodynamic profiles and its indispensable role for the understanding and the simulation of water and energy cycles. Rev. Geophys., 53, 819-895, 2015. 